OPEN ACCESS

Edited by:

Jun Chen,

University of Science and Technology

Beijing, China

Reviewed by:

Fengxia $\mathrm{Hu}$,

Institute of Physics (CAS), China

Hongfei Liu,

Yangzhou University, China

*Correspondence:

Ying Sun

sunying@buaa.edu.cn

Specialty section:

This article was submitted to Physical Chemistry and Chemical

Physics,

a section of the journal

Frontiers in Chemistry

Received: 09 December 2017

Accepted: 18 January 2018

Published: 06 February 2018

Citation:

Zhao W, Sun Y, Liu Y, Shi K, Lu H,

Song $P$, Wang $L$, Han $H$, Yuan $X$ and

Wang C (2018) Negative Thermal

Expansion over a Wide Temperature

Range in Fe-Doped MnNiGe

Composites. Front. Chem. 6:15.

doi: 10.3389/fchem.2018.00015

\section{Negative Thermal Expansion over a Wide Temperature Range in Fe-Doped MnNiGe Composites}

\author{
Wenjun Zhao ${ }^{1}$, Ying Sun ${ }^{1 *}$, Yufei Liu ${ }^{2}$, Kewen Shi ${ }^{1}$, Huiqing Lu ${ }^{1}$, Ping Song ${ }^{1}$, Lei Wang ${ }^{1}$, \\ Huimin Han ${ }^{1}$, Xiuliang Yuan ${ }^{1}$ and Cong Wang ${ }^{1}$ \\ ${ }^{1}$ Department of Physics, Center for Condensed Matter and Materials Physics, Beihang University, Beijing, China, ${ }^{2}$ Capital \\ Normal University High School, Beijing, China
}

Fe-doped MnNiGe alloys were successfully synthesized by solid-state reaction. Giant negative thermal expansion (NTE) behaviors with the coefficients of thermal expansion (CTE) of $-285.23 \times 10^{-6} \mathrm{~K}^{-1}(192-305 \mathrm{~K})$ and $-1167.09 \times 10^{-6} \mathrm{~K}^{-1}(246-305 \mathrm{~K})$ have been obtained in $\mathrm{Mn}_{0.90} \mathrm{Fe}_{0.10} \mathrm{NiGe}$ and $\mathrm{MnNi}_{0.90} \mathrm{Fe}_{0.10} \mathrm{Ge}$, respectively. Furthermore, these materials were combined with $\mathrm{Cu}$ in order to control the NTE properties. The results indicate that the absolute value of CTE gradually decreases with increasing $\mathrm{Cu}$ contents. In $\mathrm{Mn}_{0.92} \mathrm{Fe}_{0.08} \mathrm{NiGe} / x \% \mathrm{Cu}$, the CTE gradually changes from $-64.92 \times 10^{-6}$ $\mathrm{K}^{-1}(125-274 \mathrm{~K})$ to $-4.73 \times 10^{-6} \mathrm{~K}^{-1}(173-229 \mathrm{~K})$ with increasing value of $x$ from 15 to 70. The magnetic measurements reveal that the NTE behaviors in this work are strongly correlated with the process of the magnetic phase transition and the introduction of $\mathrm{Fe}$ atoms could also change the spiral anti-ferromagnetic (s-AFM) state into ferromagnetic (FM) state at low temperature. Our study launches a new candidate for controlling thermal expansion properties of metal matrix materials which could have potential application in variable temperature environment.

Keywords: Fe-Doped MnNiGe, Heusler alloys, negative thermal expansion, magnetic transition, composites

\section{INTRODUCTION}

Solid materials usually expand during heating while contract during cooling, i.e., positive thermal expansion (PTE). When functional materials are used in variable temperature environment, PTE will reduce the structural stability and safety reliability of precision parts, or even destroy the functional properties of materials (Chen et al., 2015). Thus, zero thermal expansion (ZTE) or controllable thermal expansion materials are highly required and also wildly applied (Wang et al., 2012) in areas such as aerospace, optical instruments, high-precision machining and processing, microelectronic devices, electro-optical sensor, electronic packaging, and so on. It is important to explore new NTE materials, which could be used to combine with PTE materials as thermalexpansion compensators to obtain near-ZTE materials by adjusting their mass ratio. Until now, there are some kinds of material systems with NTE property have been reported, such as $\mathrm{ZrW}_{2} \mathrm{O}_{8}$ (Sun X. J. et al., 2013; Ge et al., 2016), $\mathrm{PbTiO}_{3}$-based compounds (Chen et al., 2011), (Bi, $\left.\mathrm{La}\right) \mathrm{NiO}_{3}$ (Azuma et al., 2011), antiperovskite manganese nitrides (Sun et al., 2012b; Yan et al., 2012), $\mathrm{ScF}_{3}$-based compounds (Greve et al., 2010), $\mathrm{La}(\mathrm{Fe}, \mathrm{Co}, \mathrm{Si})_{13}$ (Huang et al., 2013), MnCoGe-based materials (Zhao et al., 2015), $\mathrm{Ca}_{2} \mathrm{RuO}_{4}$ (Takenaka et al., 2017), and so on. The previous studies have revealed different mechanisms which lead to the NTE behaviors, including phonon model (Li et al., 2002), rigid unit modes (Dove et al., 1995), phase transition mechanism (Xing et al., 2003), 
electronic valence transfer mechanism (Arvanitidis et al., 2003), magnetic transition mechanism (Sun et al., 2012a), and so on.

Recently, MnM'X (M': Co, Ni, Fe, et al, X: Si, Ge, Sn, et al) Heusler alloys have attracted a lot of attention due to the abundant structural and magnetic properties. In 2013, researchers found that the volume of polycrystalline $\mathrm{MnCoSi}$ alloy contracted about $0.2 \%$ at $300 \mathrm{~K}$ (Barcza et al., 2013). Especially, Zhao et al. (2015) found that $\mathrm{MnCo}_{0.98} \mathrm{Cr}_{0.02} \mathrm{Ge}$, bonded with 3-4 wt.\% epoxy, undergoes a giant NTE with a linear thermal coefficient of $-119 \times 10^{-6} \mathrm{~K}^{-1}$ from 250 to $305 \mathrm{~K}$ during the martensitic structural transition. Furthermore, the result from Lin et al. (2016) indicates that fine-powder $\mathrm{Mn}_{0.98} \mathrm{CoGe}$ can broaden the NTE temperature window to $\sim 135 \mathrm{~K}(258-393 \mathrm{~K})$ with CTE $-79.6 \times 10^{-6} \mathrm{~K}^{-1}$.

As a typical MnM'X Heusler alloy, MnNiGe undergoes a martensitic structural transition from the hexagonal $\mathrm{Ni}_{2} \mathrm{In}$ type structure (space group P63/mmc, 194) to orthorhombic TiNiSi-type structure (space group Pnma, 62 ) at $\sim 470 \mathrm{~K}$ during cooling process (Ma et al., 2015), which means that there is a potentially good negative thermal expansion effect in this system. Otherwise, the previous study indicates that the introduction of $\mathrm{Fe}$ in $\mathrm{MnNiGe}$ alloy can change the magnetic competing relationship in parent phases (Liu et al., 2012). And researchers do have observed NTE property in MnNiGe-based compounds after combining with epoxy resin, i.e., a giant CTE of -60.7 $\times 10^{-6} \mathrm{~K}^{-1}$ over $231-338 \mathrm{~K}(\Delta \mathrm{T}=107 \mathrm{~K})$ and a near-ZTE of $0.6 \times 10^{-6} \mathrm{~K}^{-1}$ over $175-231 \mathrm{~K}(\Delta \mathrm{T}=56 \mathrm{~K})$ for the sample of $\mathrm{Mn}_{0.94} \mathrm{Fe}_{0.06} \mathrm{NiGe}$ after mixing with $20 \mathrm{wt} . \%$ of epoxy resin (Xu et al., 2017). Unfortunately, the NTE properties of pure $\mathrm{MnNiGe-based} \mathrm{compounds} \mathrm{have} \mathrm{not} \mathrm{been} \mathrm{reported} \mathrm{due}$ to the brittle of MnNiGe-based compounds, to our knowledge. Thus, we prepared Fe-doped MnNiGe compounds successfully by solid-state reaction, and observed the giant NTE properties near room temperature (RT) in pure parent alloys. Moreover, the NTE properties were further controlled by combining with commercial $\mathrm{Cu}$. The obtained results in this work broaden the application of $\mathrm{Fe}$-doped $\mathrm{MnNiGe}$ alloys.

\section{EXPERIMENTAL DETAILS}

Polycrystalline samples of $\mathrm{Mn}_{1-x} \mathrm{Fe}_{x} \mathrm{NiGe}(x=0.08,0.10,0.16)$ and $\mathrm{MnNi}_{0.90} \mathrm{Fe}_{0.10} \mathrm{Ge}, \mathrm{Mn}_{1-x} \mathrm{Fe}_{x} \mathrm{NiGe} / \mathrm{Cu}(x=0.08,0.10,0.16)$ and $\mathrm{MnNi}_{0.90} \mathrm{Fe}_{0.10} \mathrm{Ge} / \mathrm{Cu}$ composites (hereafter referred as MF $x$ $(x=8,10,16)$ and NF10, MFx/Cu $(x=8,10,16)$ and $\mathrm{NF} 10 / \mathrm{Cu}$, respectively) were prepared by solid-state reaction, using $\mathrm{Mn}, \mathrm{Fe}, \mathrm{Ni}, \mathrm{Ge}$, and $\mathrm{Cu}(4 \mathrm{~N})$ powders as raw materials (Deng et al., 2015). Firstly, the stoichiometric amounts of the starting materials ( $\mathrm{Mn}, \mathrm{Ni}, \mathrm{Fe}, \mathrm{Ge}$ powders) were mixed, then ground about $2 \mathrm{~h}$ in a mortar and pressed into pellets by tablet machine. The pellets were sealed in a quartz tube under vacuum $\left(10^{-5} \mathrm{~Pa}\right)$. The quartz tube was sintered in a box furnace at $900^{\circ} \mathrm{C}$ for $80 \mathrm{~h}$ and cooled down to RT. Then, the composites of $\mathrm{Mn}_{1-x} \mathrm{Fe}_{x} \mathrm{NiGe} / \mathrm{Cu}(x=0.08,0.10,0.16), \mathrm{MnNi}_{0.90} \mathrm{Fe}_{0.10} \mathrm{Ge} / \mathrm{Cu}$ were prepared by mixing $\mathrm{Mn}_{1-x} \mathrm{Fe}_{x} \mathrm{NiGe}(x=0.08,0.10,0.16)$, $\mathrm{MnNi}_{0.90} \mathrm{Fe}_{0.10} \mathrm{Ge}$ and commercial $\mathrm{Cu}$ powder with different mass ratio, respectively. After grounding the mixture in an agate mortar for $1 \mathrm{~h}$, the mixture was pressed into rectangular blocks and sealed into quartz tubes in vacuum. Finally, the quartz tubes were sintered in a box furnace at $500^{\circ} \mathrm{C}$ for $10 \mathrm{~h}$, and then cooled down to RT naturally.

$\mathrm{X}$-ray diffraction (XRD) patterns were obtained from an $\mathrm{X}$ ' Pert PRO powder diffractometer using $\mathrm{Cu} \mathrm{K}_{\alpha}$ radiation at (RT), and the XRD data were handled with a software MDI jade 6.0. The linear thermal expansion coefficients of samples were measured from 125 to $475 \mathrm{~K}$ by using a Netzsch DIL $402 \mathrm{C}$ dilatometer. The instantaneous linear coefficient of thermal expansion $\alpha_{1}$ over certain temperature range for every sample was calculated based on the as-measured thermal strain. The $\alpha_{1}$ is defined as:

$$
\alpha_{1}=\left(1 / \mathrm{L}_{0}\right)^{*}(\mathrm{dL} / \mathrm{dT})
$$

where $\mathrm{dL}$ and $\mathrm{dT}$ are the variations in length and temperature respectively, $\mathrm{L}_{0}$ is the initial length at $125 \mathrm{~K}, \mathrm{dL} / \mathrm{L}_{0}$ is the thermal expansion strain (Chen et al., 2015).

The temperature dependence of magnetization curves were measured between 10 and $450 \mathrm{~K}$ under zero field-cooling condition (ZFC) in an applied magnetic field of 1,000 Oe using a Physical Property Measurement System (PPMS). A scanning electron microscopy (SEM, NanoSEM430, FEI) was used to characterize the microstructure of composites.

\section{RESULTS AND DISCUSSION}

\section{Phase Purity and Crystal Structure}

First, the room temperature XRD patterns of MF8, MF10, MF16, and NF10 are collected to inspect the effect of Fe doping on the crystal structure of MnNiGe alloy. As shown in Figure 1, the mainly diffraction patterns of samples can be indexed using hexagonal $\mathrm{Ni}_{2}$ In-type structure. Based on XRD data, the lattice parameters $\left(a_{h}\right.$ and $\left.c_{h}\right)$ of MF8, MF10, MF16, and NF10 are calculated by Rietveld refinement as shown in Figure 1. The results indicate that both $a_{h}$ and $c_{h}$ decrease with increasing $\mathrm{Fe}$ doping content at $\mathrm{Mn}$ sites because the atom radius of the $\mathrm{Mn}$ is larger than Fe. From the enlarged view of XRD patterns in the inset of Figure 1, it is clear that residual orthorhombic TiNiSitype structure appeared in the main matrix of hexagonal $\mathrm{Ni}_{2} \mathrm{In}$ type structure for all four samples, the residual mass fractions of orthorhombic phase are calculated to be $14,4,4$, and $3 \mathrm{wt} . \%$, respectively.

\section{Thermal Expansion Properties}

Linear thermal expansion curves of samples have been recorded from 125 to $475 \mathrm{~K}$ in Figure 2. Table 1 lists the thermal expansion values and the related temperature ranges. As shown in Figure 2A, obvious NTE behaviors are observed in MF10 and NF10 near RT, respectively. The average CTE of MF10 reaches to a giant value of $-285.23 \times 10^{-6} \mathrm{~K}^{-1}$ with a wide operationtemperature range of $192-305 \mathrm{~K}(\Delta \mathrm{T}=113 \mathrm{~K})$, while the CTE of NF10 is as large as $-1167.09 \times 10^{-6} \mathrm{~K}^{-1}$ with a temperature range of $246-305 \mathrm{~K}(\Delta \mathrm{T}=59 \mathrm{~K})$. The results indicate that Fedoped at $\mathrm{Ni}$ sites in $\mathrm{MnNiGe}$ has a broader temperature range while a larger CTE value can be obtained by Fe doping at Mn sites. Unfortunately, the $\mathrm{dL} / \mathrm{L}_{0}$ curves of pure MF8 and MF16 

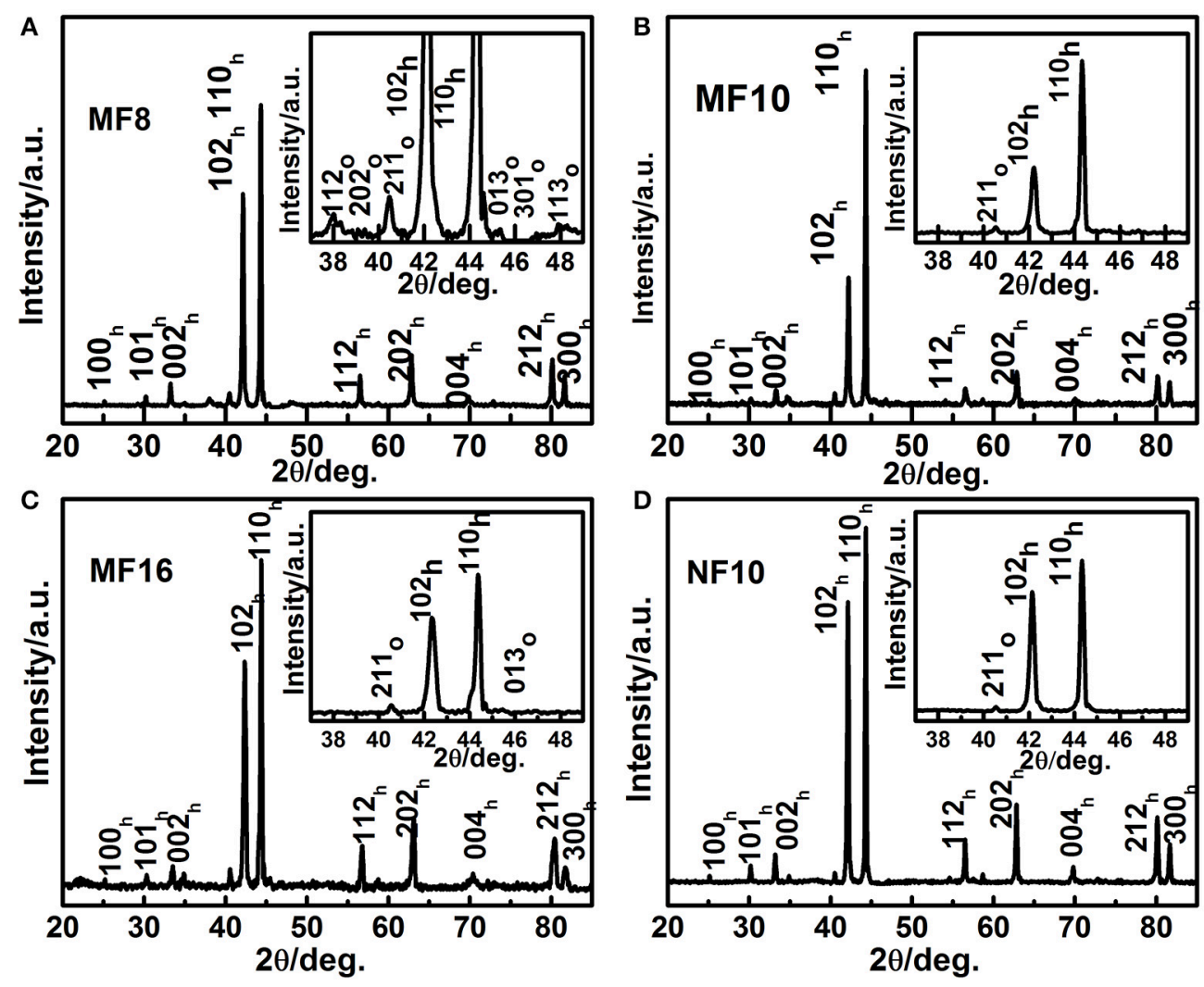

FIGURE 1 | Room temperature X-ray diffraction patterns of (A) MF8, (B) MF10, (C) MF16, and (D) NF10.

have not been obtained due to the brittle of the two samples. By comparison, the CTE of Fe-doping MnNiGe is obviously larger than the reported systems such as $\mathrm{ZrW}_{2} \mathrm{O}_{8}\left(-9.1 \times 10^{-6} \mathrm{~K}^{-1}\right)$ (Mary et al., 1996), MnCoGe-based compound $\left(-141 \times 10^{-6}\right.$ $\mathrm{K}^{-1}$ ) (Zhao et al., 2015; Lin et al., 2016), and Mn-Co-Ge-In compound $\left(-51.5 \times 10^{-6} \mathrm{~K}^{-1}\right)$ (Shen et al., 2017).

One of the typical applications for NTE materials is to control thermal expansion behaviors in the form of PTE/NTE composites. In this work, commercial $\mathrm{Cu}$ has been selected as matrix metal because of its excellent electronic and mechanic properties and wide usage in the electronic industrial area. Otherwise, in our materials, Fe-doped MnNiGe alloys always collapsed during naturally cooling or the measurement process of $\mathrm{CTE}$, the combination with $\mathrm{Cu}$ can also increase the mechanical property of Fe-doped $\mathrm{MnNiGe}$ alloys. Therefore, variable mass ratio of $\mathrm{MF} 8 / x \% \mathrm{Cu}(x=15,25,30,40,50,70,85,100)$, $\mathrm{MF} 16 / x \% \mathrm{Cu}(x=10,20,30), \mathrm{MF} 10 / 35 \% \mathrm{Cu}$ and $\mathrm{NF} 10 / 35 \% \mathrm{Cu}$ compounds were prepared. Figure 2D displays the XRD patterns of the $\mathrm{MF} 8 / x \% \mathrm{Cu}$ composites. All of the diffraction peaks can be indexed as characteristic peaks of either MF8 or $\mathrm{Cu}$ and no additional peaks were observed, indicating that no chemical reaction generated between MF8 and $\mathrm{Cu}$ during heating treatment in furnace.

The measurements for linear thermal expansion behaviors have been carried out as shown in Figure 2. The amplitudes of the
NTE behaviors $\left(\Delta \mathrm{L} / \mathrm{L}_{0}\right)$ in MF10 and NF10 obviously decrease from 3.18 to $0.40 \%$ and 6.95 to $0.32 \%$ by introducing $35 \% \mathrm{Cu}$, and the CTEs (listed in Table 1) gradually decrease from $-285.23 \times$ $10^{-6} \mathrm{~K}^{-1}(192-305 \mathrm{~K})$ and $-1167.09 \times 10^{-6} \mathrm{~K}^{-1}(246-305 \mathrm{~K})$ to $-26.15 \times 10^{-6} \mathrm{~K}^{-1}(167-290 \mathrm{~K})$ and $-56.73 \times 10^{-6} \mathrm{~K}^{-1}$ $(176-247 \mathrm{~K})$, respectively, as shown in Figure 2A. The linear thermal expansion behaviors of $\mathrm{MF} 8 / x \% \mathrm{Cu}$ and $\mathrm{MF} 16 / x \% \mathrm{Cu}$ are presented in Figures 2B,C). With the increasing of $\mathrm{Cu}$ mass ratio $x$ for both of the two serials composites, the NTE temperature ranges become narrow, i.e., the decreasing of the $\Delta \mathrm{T}$, and the absolute CTE values increase. Obviously, in $\mathrm{MF} 8 / x \% \mathrm{Cu}$ serials composites, a low thermal expansion behavior with a CTE of $-4.73 \times 10^{-6} \mathrm{~K}^{-1}$ in a wide temperature range $173-229 \mathrm{~K}$ $(\Delta \mathrm{T}=56 \mathrm{~K})$ is observed in $\mathrm{MF} 8 / 70 \% \mathrm{Cu}$ composite. When the mass ratio of $\mathrm{Cu}$ is $30 \%$ (sample No. 7 in Table 1), the CTE is $-41.99 \times 10^{-6} \mathrm{~K}^{-1}$ between $126 \mathrm{~K}$ to $270 \mathrm{~K}$. The NTE behavior is observed in the temperature range $119-274 \mathrm{~K}(\Delta \mathrm{T}=155 \mathrm{~K})$ for sample $\mathrm{MF} 8 / 15 \% \mathrm{Cu}$, and the corresponding CTE is -64.92 $\times 10^{-6} \mathrm{~K}^{-1}$. In $\mathrm{MF} 16 / 10 \% \mathrm{Cu}$ composite, there is a low CTE of $1.16 \times 10^{-6} \mathrm{~K}^{-1}$ in a wide temperature range $125-215 \mathrm{~K}$ $(\Delta \mathrm{T}=90 \mathrm{~K})$. With further increasing temperature, a sharp decreasing in NTE curve is observed and the corresponding CTE is $-174.58 \times 10^{-6} \mathrm{~K}^{-1}(216-228 \mathrm{~K})$. However, the negative thermal expansion disappeared when the mass ratio of $\mathrm{Cu}$ increases to $30 \%$ in $\mathrm{MF} 16 / x \% \mathrm{Cu}$ composites, this may be due 

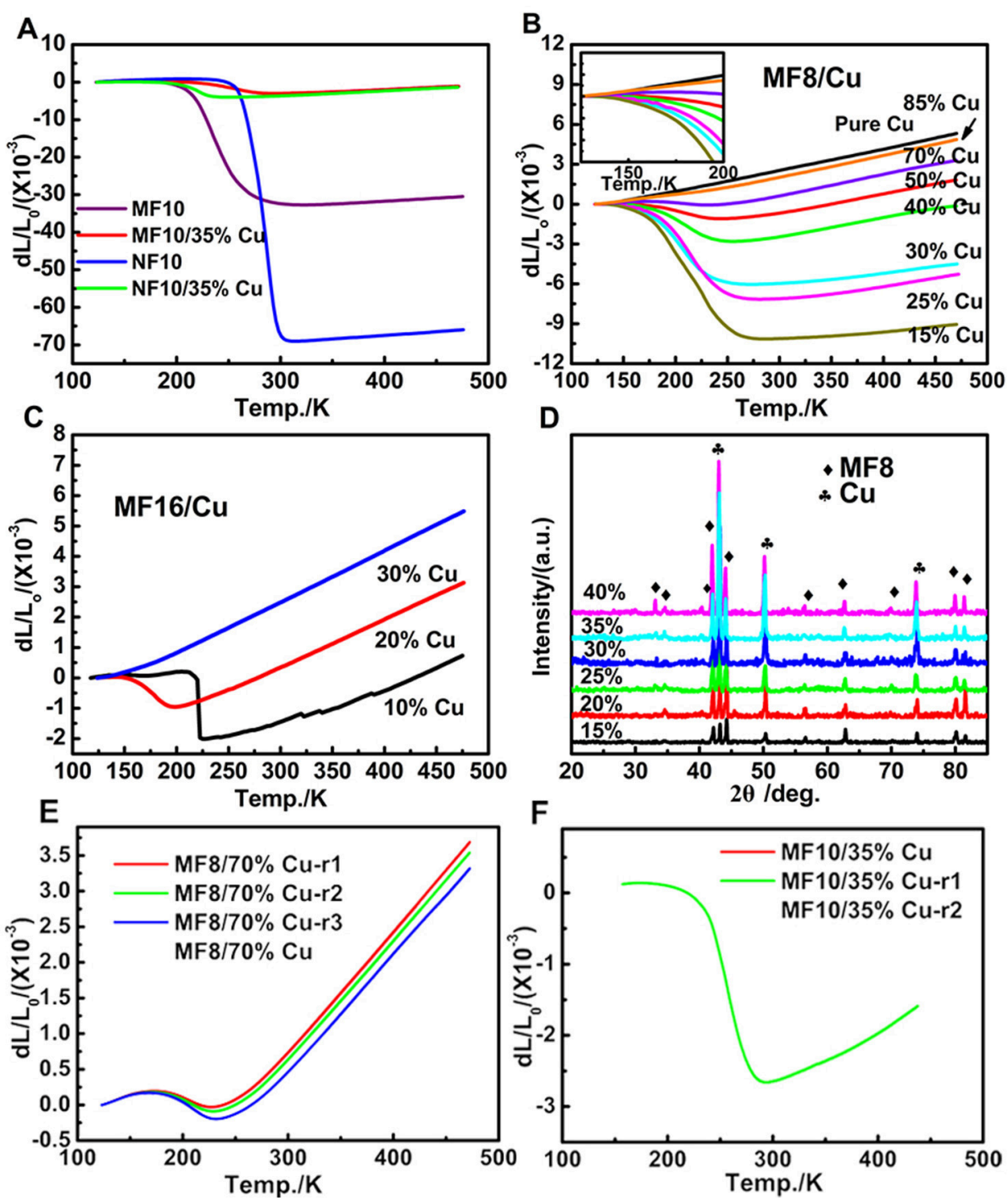

FIGURE 2 | The temperature dependence of $d \mathrm{~L} / \mathrm{L}_{0}$ curves of (A) MF10, NF10, MF10/35\%Cu and NF10/35\%Cu, (B) MF8/ $x \% \mathrm{Cu}(x=15,25,30,40,50,70,85,100)$ and (C) $M F 16 / x \% \mathrm{Cu}(x=10,20,30)$, where the $\mathrm{L}_{0}$ is the length of the sample at $123 \mathrm{~K}$; (D) $\mathrm{X}$-Ray patterns of MF8/ $\mathrm{x} \% \mathrm{Cu}$ composites at RT; the repeated measurement temperature dependence of $d L / L_{0}$ curves of $(E) M F 8 / 70 \% \mathrm{Cu}$ and $(\mathbf{F}) \mathrm{MF} 10 / 35 \% \mathrm{Cu}$.

to the NTE temperature region decreases to below $125 \mathrm{~K}$ which is the minimum measuring temperature limit. In order to confirm the stability of the NTE behavior in our samples, we measured the thermal expansion properties of $\mathrm{MF} 10 / 35 \% \mathrm{Cu}$ and $\mathrm{MF} 8 / 70 \% \mathrm{Cu}$ again and the results are very close to the first measurement result, as shown in Figures 2E,F. Therefore, our studies suggest this could be a new alternative for metal matrix composites to achieve near-ZTE materials.

\section{Magnetic Properties}

To well understand the thermal expansion property of samples, magnetic properties are further investigated. Temperature dependence of magnetization (M-T) curves of the Fe-doped $\mathrm{MnNiGe}$ alloys are first carried out as shown in Figure 3A (the inset presents the breaking part of MF16), and the related dM/dT-T curves are shown in Figure 3B. The M-T curves of MF8, MF10, and NF10 increase slowly first and then drop sharply with temperature increasing, indicating that a magnetic transition occurred. With further increasing temperature, the M-T curves undergo another sharp drop and hence enter into paramagnetic phases (PM). There are two transition points of magnetic properties, which indicates two different ordered magnetic phases exist in MF8, MF10, and NF10. The magnetic transition temperature points (the low transition temperature $T_{t}$ and Néel temperature $T_{N}$ ) are defined in Figure 3B (Azuma et al., 2011) to be $\mathrm{T}_{\mathrm{t}}=158 \mathrm{~K}, 146 \mathrm{~K}$ and $112 \mathrm{~K}, \mathrm{~T}_{\mathrm{N}}=272,224$, and $218 \mathrm{~K}$ for MF8, MF10, and NF10, respectively. 
In order to clarify the types of magnetic phases, the isothermal magnetization $\mathrm{M}(\mathrm{H})$ curves at $10 \mathrm{~K}$ of $\mathrm{MF} 10$ and $\mathrm{NF} 10$ are carried out in Figure 3C. The M-H curves of MF10 and NF10 tend to be linear behavior with no hysteresis behaviors and near zero macroscopic saturation magnetization in the low magnetic field, indicating typical AFM characters (Song et al., 2008; Sun Y. et al., 2013). The slightly bending of the M-H curves may be caused by a potential magnetic phase transition under external magnetic field. Depending on the previous studies,

TABLE 1 | CTEs and $\triangle T$ of the composites with different Cu mass ratio.

\begin{tabular}{|c|c|c|c|c|}
\hline No. & Samples & $\begin{array}{l}\text { Mass ratio of } \\
\text { MFx/NF10 (\%) }\end{array}$ & CTE $\left(\times 10^{-6} \mathrm{~K}^{-1}\right)$ & $\begin{array}{l}\text { Temperature } \\
\text { range }(\Delta T) / K\end{array}$ \\
\hline 1 & MF10 & 100 & -285.23 & $192-305$ (113) \\
\hline 2 & MF10/35\%Cu & 65 & -26.15 & $167-290(123)$ \\
\hline 3 & NF10 & 100 & -1167.09 & 246-305 (59) \\
\hline 4 & $\mathrm{NF} 10 / 35 \% \mathrm{Cu}$ & 65 & -56.73 & $176-247(71)$ \\
\hline 5 & MF8/15\%Cu & 85 & -64.92 & $125-274(149)$ \\
\hline 6 & MF8/25\%Cu & 75 & -50.23 & $125-264(140)$ \\
\hline 7 & MF8/30\%Cu & 70 & -41.99 & $126-270$ (144) \\
\hline 8 & MF8/40\%Cu & 60 & -22.53 & 130-255 (125) \\
\hline 9 & MF8/50\%Cu & 50 & -11.32 & $141-241(100)$ \\
\hline 10 & MF8/70\%Cu & 30 & -4.73 & $173-229(56)$ \\
\hline \multirow[t]{2}{*}{11} & MF16/10\%Cu & 90 & 1.16 & $125-215(90)$ \\
\hline & & & -174.58 & $216-228(112)$ \\
\hline 12 & MF16/20\%Cu & 80 & -13.26 & 125-195 (71) \\
\hline
\end{tabular}

this AFM phase in low temperature is the typical spiral AFM (s-AFM) (Johnson and Frederick, 1973). In MF10 and NF10, with temperature increasing to $\mathrm{T}_{t}, \mathrm{~s}$-AFM phase transforms to another AFM phase, of which the magnetization is lower than that of s-AFM, as shown in Figure 3A. With further increasing temperature, all of the three samples transit to PM phase at $\mathrm{T}_{\mathrm{N}}$, successively. However, only one magnetic phase transition is observed in MF16, and the M-H curve shows typical FM characteristics which indicates the magnetic transition of MF16 is a FM-PM transition at Currie temperature $\mathrm{T}_{\mathrm{C}}=128 \mathrm{~K}$ as shown in Figure 3B. In addition, in $\operatorname{MF} x(x=8,10,16)$, with Fe content increasing, the magnetization increased (Figure $3 \mathbf{A}$ ) and $\mathrm{T}_{\mathrm{t}}$ or $\mathrm{T}_{\mathrm{N}} / \mathrm{T}_{\mathrm{C}}$ decreased regularly (Figure $3 \mathrm{~B}$ ), signifying that the introduction of Fe has changed the s-AFM structure into an FM state. Moreover, Figure 3D shows the relationship of $\mathrm{dL} / \mathrm{dL}_{0}$ and $\mathrm{M}(\mathrm{T})$ curves in MF10 and NF10. The black real line and the black dot real line are $\mathrm{dL} / \mathrm{dL}_{0}$ curve and $\mathrm{M}(\mathrm{T})$ curve for MF10, while the temperature range related NTE property has been defined by the perpendicular black dot dash line with a double arrow. Similarly, the red ones are corresponding to NF10. It is clear that the large temperature windows of observed NTE behaviors are in consistent with the magnetization decreasing slowly during the AFM-PM transformation in MF10 and NF10, which indicates a strong relationship between magnetism and thermal expansion properties in these serials compounds, as well the composites.

\section{Microstructural Properties}

The microstructures of pure MF10 and NF10, composites $\mathrm{MF} 8 / 70 \% \mathrm{Cu}$ and $\mathrm{MF} 10 / 35 \% \mathrm{Cu}$ are carried out and shown in
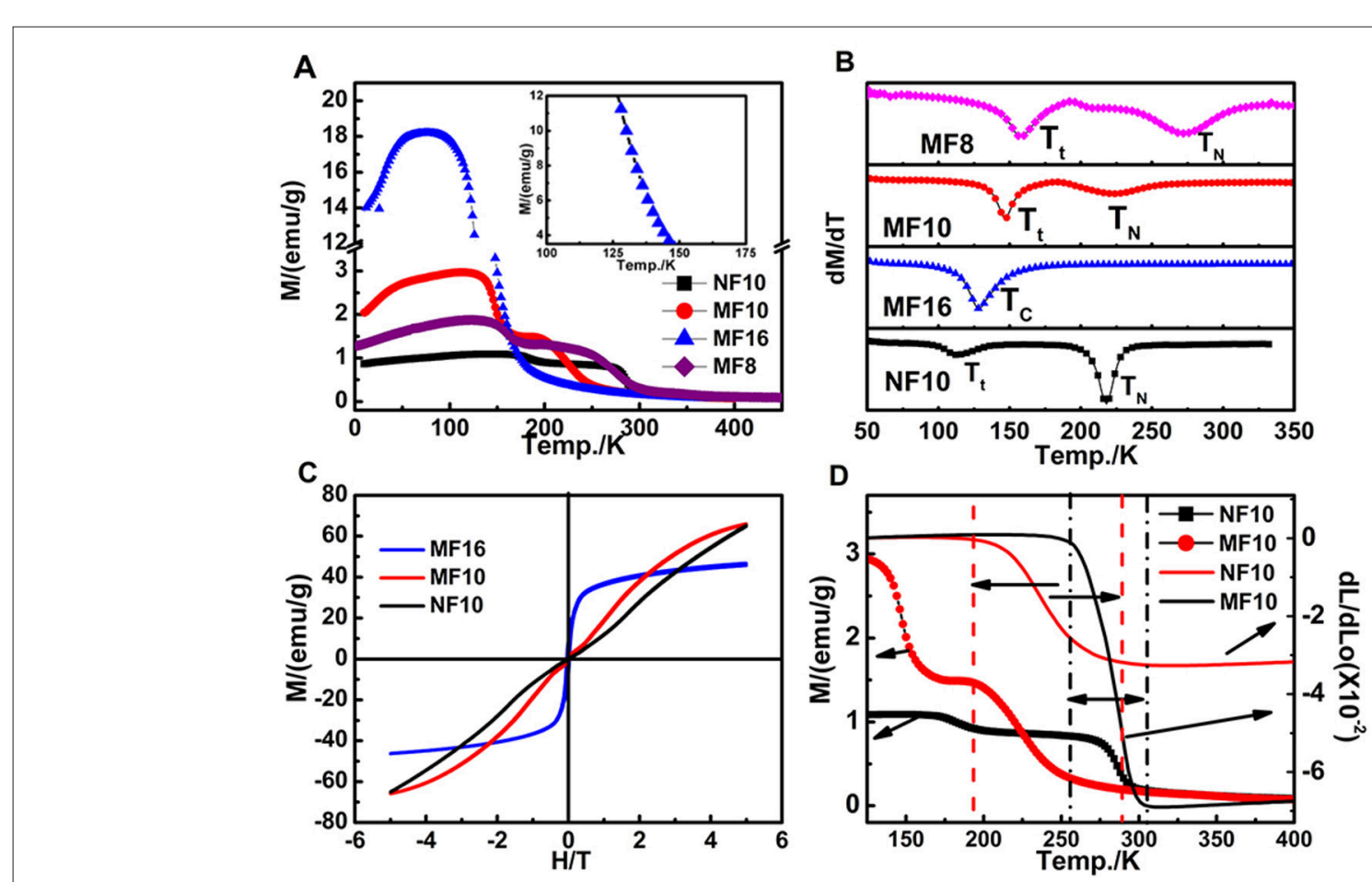

FIGURE 3 | (A) M-T curves (ZFC) of MF8, MF10, MF16, and NF10 with external magnetic field of 1000 Oe; (B) the related dM/dT-T curves of four samples; (C) the isothermal $\mathrm{M}(\mathrm{H})$ curves of MF10, MF16, and NF10 at $10 \mathrm{~K}$; (D) M(T) curves and dL/Lo curves of pure MF10 and NF10 over $125-400 \mathrm{~K}$. 
Figure 4. Firstly, in both of MF10 and NF10 samples have massive cavities, and the size of cavities in MF10 is smaller $(\sim 28 \mu \mathrm{m})$ than that in NF10 in which the size of cavities is as large as $60 \mu \mathrm{m}$. Compared with the results of NTE in Figure 2A, large size of cavities sample (NF10) has a larger NTE behavior which indicated that the porosity in pure Fedoped MnNiGe has positive contribution to the large NTE behaviors. Otherwise, it is clear that two kinds of aggregations and a few holes and cracks can be seen in composites samples. One aggregation consists of small flaky particles with a size $\sim 5 \mu \mathrm{m}$, and the other one consists of irregular and granular particles with a larger size (more than $20 \mu \mathrm{m}$ ). Meanwhile, the chemical compositions of these areas are also analyzed by the energy dispersive X-Ray spectroscopy (EDS). We labeled the locations containing different species in Figures 5A,D as areas 1 and 2, areas 3 and 4, respectively. The related EDS of areas 12 and areas 3-4 are shown in Figures 5B,C and Figures 5E,F, respectively. As shown in Figure 5, $\mathrm{Mn}, \mathrm{Ni}, \mathrm{Fe}, \mathrm{Ge}$, and $\mathrm{Cu}$ peaks are observed in all of the spectra. In $\mathrm{MF} 8 / 70 \% \mathrm{Cu}$, the composition analysis demonstrates that the atomic ratio of area 1 is $\mathrm{Mn}$ : Fe: Ni: Ge: $\mathrm{Cu}=24.36$ : 3.77: 35.70: 32.95: 3.22. Combined with Figure 2D, the main component of area 1 is MF8, the small amount of $\mathrm{Cu}$ is mainly originating from the adherence during mixed grinding and isothermal heat treatment. The atomic ratio of the area 2 is $\mathrm{Mn}$ : Fe: Ni: Ge: $\mathrm{Cu}=2.01: 0.22: 0.83: 1.30: 95.62$, which indicates the major component of the particles in area 2 is $\mathrm{Cu}$ metal. Using the same analysis method, the main component of area 3 is MF10, while the component of area 4 is mainly $\mathrm{Cu}$, as shown in Figures 5E,F. It can be found that the cracks and holes are filled with $\mathrm{Cu}$ particles and the composites have a more compact structure with increasing $\mathrm{Cu}$ content, i.e., the MF8 is well dispersed in $\mathrm{Cu}$ matrix in $\mathrm{MF} 8 / 70 \% \mathrm{Cu}$, where the stability of the composites is guaranteed (Yan et al., 2014). The composites
A

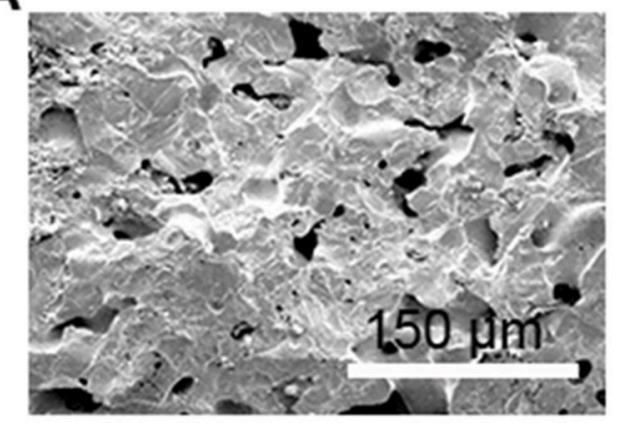

C

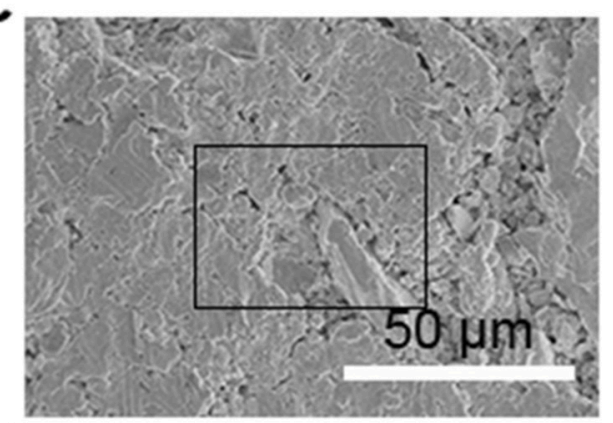

E

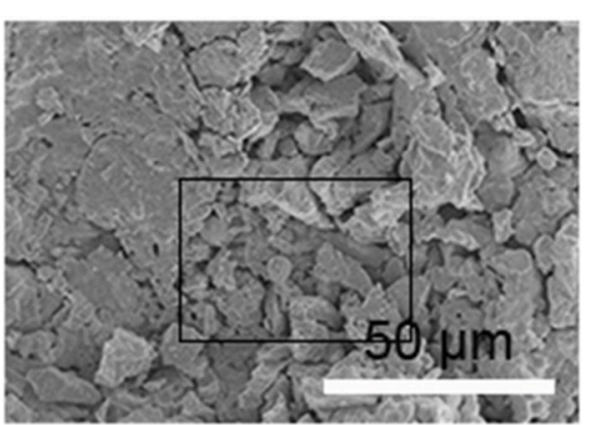

B

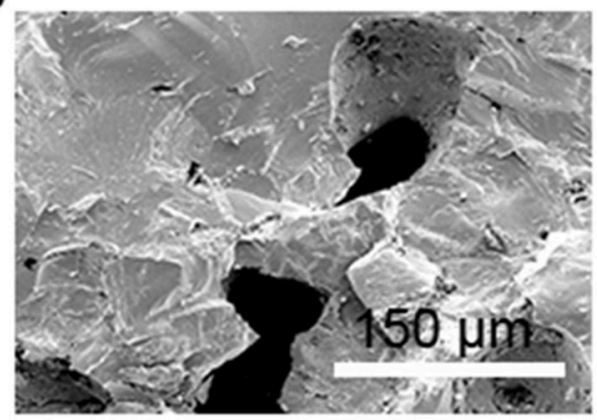

D

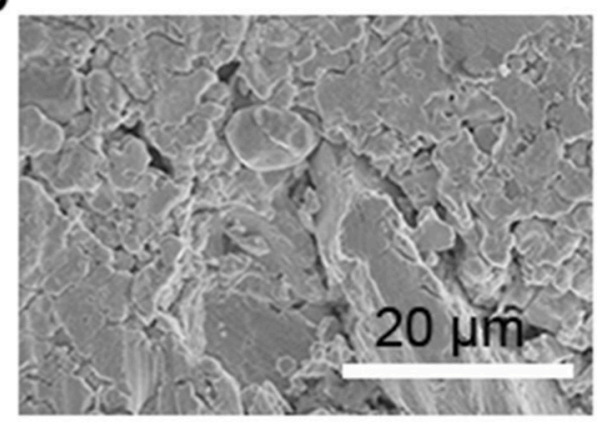

F

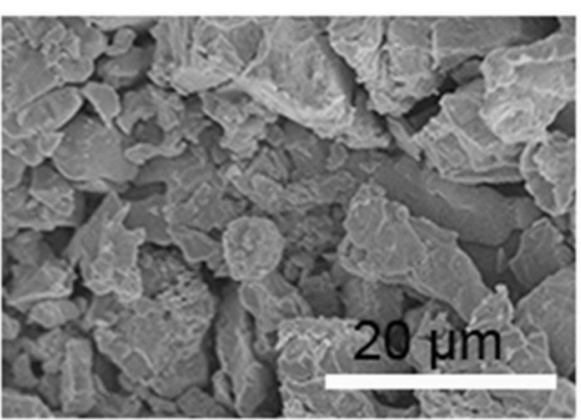

FIGURE 4 | SEM images of (A) MF10 and (B) NF10, (C,D) MF8/70\%Cu, (E,F) MF10/35\%Cu. 

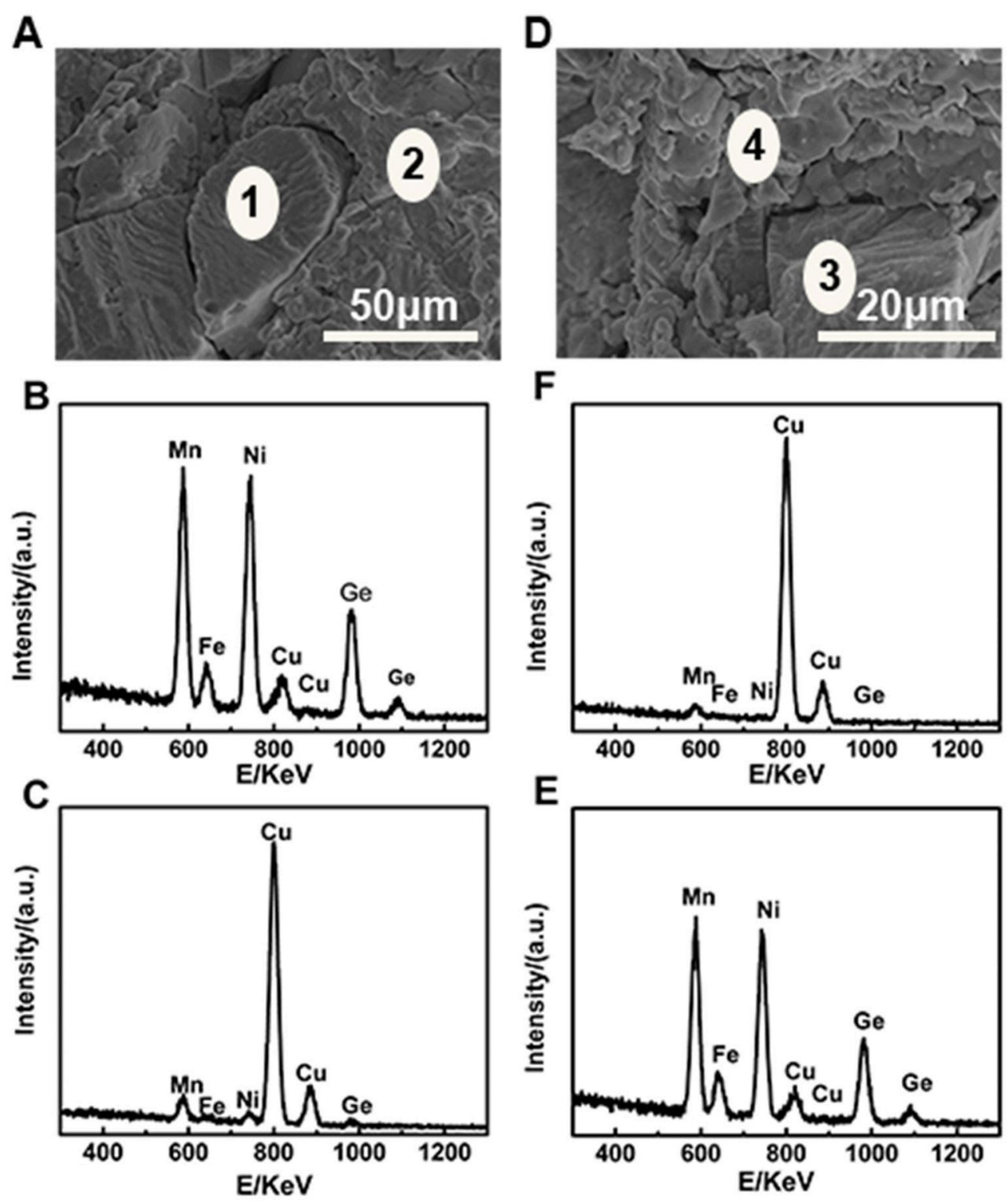

FIGURE 5 | SEM images of (A) MF8/70\%Cu and (D) MF10/35\%Cu, respectively and related EDS spectra of (B,C) in (A) and (E,F) in (D), respectively.

display low thermal expansion or near-ZTE properties due to the competition between the positive expansion property of $\mathrm{Cu}$ matrix and the negative thermal expansion properties of Fedoped $\mathrm{MnNiGe}$ alloys when placed in a variable temperature environment.

\section{CONCLUSION}

In summary, polycrystalline samples of $\mathrm{Mn}_{1-x} \mathrm{Fe}_{x} \mathrm{NiGe}$ $(x=0.08,0.10,0.16)$ and $\mathrm{MnNi}_{0.90} \mathrm{Fe}_{0.10} \mathrm{Ge}$ were prepared by solid-state reaction. Giant negative thermal expansion properties were observed over a wide temperature region in Fe-doped MnNiGe compounds. Especially, the average coefficient of thermal expansion (CTE) for NF10 reaches as large as -1167.09 $\times 10^{-6} \mathrm{~K}^{-1}$ between 246 and $305 \mathrm{~K}(\Delta \mathrm{T}=59 \mathrm{~K})$. The scanning electron microscopy results show that the porosity has positive contribution to the NTE behavior of MF10 and NF10. AFM-PM phase transition, which is correlated with negative thermal expansion (NTE) of the samples, has been characterized by the magnetic measurements. The $\mathrm{Mn}_{1-x} \mathrm{Fe}_{x} \mathrm{NiGe} / \mathrm{Cu}(x=8$, 16) composites with controllable thermal expansion have been successfully prepared by adjusting the mass ratio of $\mathrm{Cu}$. In $\mathrm{MF} 8 / x \% \mathrm{Cu}$ composites, the absolute value of average CTE gradually decreases with increasing matrix $\mathrm{Cu}$ content and the CTE reaches to $-4.73 \times 10^{-6} \mathrm{~K}^{-1}(173-229 \mathrm{~K})$ with $70 \% \mathrm{Cu}$. In addition, the combining with metal $\mathrm{Cu}$ also modifies the mechanical performance of Fe-doped $\mathrm{MnNiGe}$ 
compounds. This can be a new candidate for controlling thermal expansion properties of metal matrix materials which may have potential applications in variable temperature environment.

\section{AUTHOR CONTRIBUTIONS}

YS, CW, WZ conceived the idea and designed experiments; WZ, YL, XY, and HH carried out experiments; and KS, HL, PS, and LW analyzed experimental results. All authors wrote and reviewed the manuscript.

\section{REFERENCES}

Arvanitidis, J., Papagelis, K., Margadonna, S., Prassides, K., and Fitch, A. N. (2003). Temperature-induced valence transition and associated lattice collapse in samarium fulleride. Nature 425, 599-602. doi: 10.1038/nature 01994

Azuma, M., Chen, W. T., Seki, H., Czapski, M., Olga, S., Oka, K., et al. (2011). Colossal negative thermal expansion in $\mathrm{BiNiO}_{3}$ induced by intermetallic charge transfer. Nat. Commun. 2:347. doi: 10.1038/ncomm s1361

Barcza, A., Gercsi, Z., Michor, H., Suzuki, K., Kockelmann, W., Knight, K. S., et al. (2013). Magnetoelastic coupling and competing entropy changes in substituted CoMnSi metamagnets. Phys. Rev. B 87:064410. doi: 10.1103/PhysRevB.87.064410

Chen, J., Hu, L., Deng, J., and Xing, X. (2015). Negative thermal expansion in functional materials: controllable thermal expansion by chemical modifications. Chem. Soc. Rev. 44, 3522-3567. doi: 10.1039/c4cs0 $0461 \mathrm{~b}$

Chen, J., Nittala, K., Forrester, J. S., Jones, J. L., Deng, J. X., Yu, R. B., et al. (2011). The role of spontaneous polarization in the negative thermal expansion of tetragonal $\mathrm{PbTiO}_{3}$-based compounds. J. Am. Chem. Soc. 133, 11114-11117. doi: $10.1021 /$ ja2046292

Deng, S. H., Sun, Y., Wu, H., Huang, Q. Z., Yan, J., Shi, K. W., et al. (2015). Invarlike behavior of antiperovskite $\mathrm{Mn}_{3+\mathrm{x}} \mathrm{Ni}_{1-\mathrm{x}} \mathrm{N}$ compounds. Chem. Mater. 27, 2495-2501. doi: 10.1021/cm504702m

Dove, M. T., Heine, V., and Hammonds, K. D. (1995). Rigid unit modes in framework silicates. Mineral. Mag. 59, 629-639. doi: 10.1180/minmag.1995.059.397.07

Ge, X. H., Liu, X. S., Cheng, Y. G., Yuan, B. H., Chen, D. X., Chao, M. J., et al. (2016). Negative thermal expansion and photoluminescence properties in a novel material $\mathrm{ZrScW}_{2} \mathrm{PO}_{12}$. J. Appl. Phys. 120:205101. doi: 10.1063/1.49 68546

Greve, B. K., Martin, K. L., Lee, P. L., Chupas, P. J., Chapman, K. W., and Wilkinson, A. P. (2010). Pronounced negative thermal expansion from a simple structure: cubic $\mathrm{ScF}_{3}$. J. Am. Chem. Soc. 132, 15496-15498. doi: $10.1021 /$ ja106711v

Huang, R. J., Liu, Y. Y., Fan, W., Tan, J., Xiao, F. R., Qian, L. H., et al. (2013). Giant negative thermal expansion in $\mathrm{NaZn}_{13}$-type $\mathrm{La}(\mathrm{Fe}, \mathrm{Si}, \mathrm{Co})_{13}$ compounds. J. Am. Chem. Soc. 135, 11469-11472. doi: 10.1021/ja40 $5161 z$

Johnson, V., and Frederick, C. G. (1973). Magnetic and crystallographic properties of ternary manganese silicides with ordered Co2P structure. Phys. Status Solidi A 20, 331-335. doi: 10.1002/pssa.2210 200133

Li, J., Yokochi, A., Amos, T. G., and Sleight, A. W. (2002). Strong negative thermal expansion along the $\mathrm{O}-\mathrm{Cu}-\mathrm{O}$ linkage in $\mathrm{CuScO}_{2}$. Chem. Mater. 14, 2602-2606. doi: $10.1021 / \mathrm{cm} 011633 \mathrm{v}$

Lin, J. C., Tong, P., Zhang, K., Tong, H. Y., Guo, X. G., Yang, C., et al. (2016). Colossal negative thermal expansion with an extended temperature interval covering room temperature in finepowdered $\mathrm{Mn}_{0.98}$ CoGe. Appl. Phys. Lett. 109, 241903. doi: 10.1063/ 1.4972234

\section{ACKNOWLEDGMENTS}

This work is financially supported by the National Natural Science Foundation of China (NSFC) (Nos. 51472017, 51732001, 51572010), the Fundamental Research Funds for the Central Universities, Aeronautical Science Foundation of China, and the Project-sponsored by SRF for ROCS, SEM. The authors also thanks Professor Lai fengLi, Professor Rong jin Huang, and Doctor Wei Wang in theTechnical Institute of Physics and Chemistry, CAS, for the help at this work.

Liu, E., Wang, W., Feng, L., Zhu, W., Li, G., Chen, J., et al. (2012). Stable magnetostructural coupling with tunable magnetoresponsive effects in hexagonal ferromagnets. Nat. Commun. 3:873. doi: 10.1038/ncomm s1868

Ma, S. C., Su, Y., Yang, M., Yang, F., Huang, Y. L., Liu, K., et al. (2015). Magnetic phase transition and magnetocaloric effect in $\mathrm{Mn}-\mathrm{Fe}-\mathrm{Ni}$ Ge ribbons. J. Alloys Compd. 629, 322-325. doi: 10.1016/j.jallcom.2014. 12.250

Mary, T. A., Evans, J. S. O., Vogt, T., and Sleight, A. W. (1996). Negative thermal expansion from 0.3 to 1050 kelvin in $\mathrm{ZrW}_{2} \mathrm{O}_{8}$. Science 272, 90-92. doi: $10.1126 /$ science. 272.5258 .90

Shen, F. R., Kuang, H., Hu, F. X., Wu, H., Huang, Q. Z., Liang, F. X., et al. (2017). Ultra-low thermal expansion realized in giant negative thermal expansion materials through self-compensation. APL Mater. 5:106102. doi: $10.1063 / 1.4990481$

Song, B., Jian, J. K., Bao, H. Q., Lei, M., Li, H., Wang, G., et al. (2008). Observation of spin-glass behavior in antiperovskite $\mathrm{Mn}_{3} \mathrm{GaN}$. Appl. Phys. Lett. 92:192511. doi: $10.1063 / 1.293105$

Sun, X. J., Cheng, X. N., and Yang, J. (2013). Effect of acids on the morphology and negative thermal expansion analysis of $\mathrm{ZrW}_{2} \mathrm{O}_{8}$ powders prepared by the hydrothermal method. Ceram. Int. 39, 165-170. doi: 10.1016/j.ceramint.2012. 06.005

Sun, Y., Guo, Y., Tsujimoto, Y., Yang, J., Shen, B., Yi, W., et al. (2013). Carbon-induced ferromagnetism in the antiferromagnetic metallic host material $\mathrm{Mn}_{3} \mathrm{ZnN}$. Inorg. Chem. 52, 800-806. doi: 10.1021/ic30 19265

Sun, Y., Guo, Y. F., Wang, X. X., Tsujimoto, Y., Matsushita, Y., and Shi, Y. G. (2012a). Resistive switching phenomenon driven by antiferromagnetic phase separation in an antiperovskite nitride $\mathrm{Mn}_{3} \mathrm{ZnN}$. Appl. Phys. Lett. 100:161907. doi: $10.1063 / 1.4704664$

Sun, Y., Wang, C., Huang, Q., Guo, Y., Chu, L., Arai, M., et al. (2012b). Neutron diffraction study of unusual phase separation in the antiperovskite nitride $\mathrm{Mn}_{3} \mathrm{ZnN}$. Inorg. Chem. 51, 7232-7236. doi: 10.1021/ic30 0978x

Takenaka, K., Okamoto, Y., Shinoda, T., Katayama, N., and Sakai, Y. (2017). Colossal negative thermal expansion in reduced layered ruthenate. Nat. Commun. 8:14102. doi: 10.1038/ncomms 14102

Wang, C., Chu, L. H., Yao, Q. R., Sun, Y., Wu, M. M., Ding, L., et al. (2012). Tuning the range, magnitude, and sign of the thermal expansion in intermetallic $\mathrm{Mn}_{3}(\mathrm{Zn}, \mathrm{M})_{\mathrm{x}} \mathrm{N}(\mathrm{M}=\mathrm{Ag}$, Ge). Phys. Rev. B 85:220103(R). doi: 10.1103/PhysRevB.85. 220103

Xing, X. R., Deng, J. X., Chen, J., and Liu, G. R. (2003). Novel thermal expansion of lead titanate. Rare Met. 22, 294-297. doi: 10.1016/S1001-0521 (08)60123-0

Xu, K., Li, Z., Liu, E., Zhou, H., Zhang, Y., and Jing, C. (2017). Magnetocaloric effect and negative thermal expansion in hexagonal Fe doped MnNiGe compounds with a magnetoelastic AFM-FM-like transition. Sci. Rep. 7:41675. doi: $10.1038 /$ srep41675

Yan, J., Sun, Y., Wang, C., Chu, L. H., Shi, Z. X., Deng, S. H., et al. (2014). Study of structure of $\mathrm{Mn}_{3} \mathrm{Cu}_{0.5} \mathrm{Ge}_{0.5} \mathrm{~N} / \mathrm{Cu}$ composite with 
nearly zero thermal expansion behavior around room temperature. Scripta Mater. 84-85, 19-22. doi: 10.1016/j.scriptamat.2014.0 4.010

Yan, X. H., Liu, J. Q., Hu, Z. Y., Li, B. Y., and Cheng, X. N. (2012). Thermal expansion of anti-perovskite $\mathrm{Mn}_{3} \mathrm{Zn}_{1-\mathrm{x}} \mathrm{Sn}_{\mathrm{X}} \mathrm{N}$ compounds. Key Eng. Mater. 512-515, 890-893. doi: 10.4028/www.scientific.net/KEM.512-51 5.890

Zhao, Y. Y., Hu, F. X., Bao, L. F., Wang, J., Wu, H., Huang, Q. Z., et al. (2015). giant negative thermal expansion in bonded MnCoGe-based compounds with $\mathrm{Ni}_{2}$ in-type hexagonal structure. J. Am. Chem. Soc. 137, 1746-1749. doi: $10.1021 /$ ja510693a
Conflict of Interest Statement: The authors declare that the research was conducted in the absence of any commercial or financial relationships that could be construed as a potential conflict of interest.

Copyright (c) 2018 Zhao, Sun, Liu, Shi, Lu, Song, Wang, Han, Yuan and Wang. This is an open-access article distributed under the terms of the Creative Commons Attribution License (CC BY). The use, distribution or reproduction in other forums is permitted, provided the original author(s) and the copyright owner are credited and that the original publication in this journal is cited, in accordance with accepted academic practice. No use, distribution or reproduction is permitted which does not comply with these terms. 